\title{
Assessment of Heart Rate Variability Derived From Blood Pressure Pulse Recordings in Intensive Care Unit Patients
}

\author{
Maximiliano Mollura ${ }^{1}$, Edoardo M Polo ${ }^{1,2}$, Li-wei H Lehman ${ }^{3}$, Riccardo Barbieri ${ }^{1}$ \\ ${ }^{1}$ Politecnico di Milano, Milano, Italy \\ 2 DIAG, Sapienza University of Rome, Italy \\ ${ }^{3}$ Massachusetts Institute of Technology, Cambridge, Massachusetts
}

\begin{abstract}
The role of Pulse Rate estimated from blood pressure pulse when used as a surrogate for Heart Rate Variability (HRV) studies has been addressed under different conditions in healthy subjects. However, there is a lack of validation in studies involving patients admitted in the Intensive Care Unit (ICU). Therefore, our study aims at validating six different possible surrogates for the ECG-derived tachogram, estimated from the time interval series between successive onset $(O)$, systolic $(S)$ and diastolic $(D)$ fiducial points extracted from arterial blood pressure $(A B P)$ and photoplethysmogram (PPG) waveforms. The validation is performed by looking at the ability of such surrogates in providing comparable estimates of the most common $H R V$ measures. Results show a high agreement between the ECG-derived and the ABP/PPG-derived series, with small biases. Results from sub-populations of patients that showed increases (and decreases) in such measures show a good ability of these surrogates in tracking autonomic changes. In addition, differently from $P P G_{O}$ and $P P G_{S}$, ventilated and sedated subjects did not show differences in estimating $H F$ power from $P P G_{D}$, indicating diastolic time intervals as less affected by such procedures.

In conclusion, HRV measures estimated from ABP or $P P G$ can be reasonably used also in studies on ICU patients whenever ECG recordings are not available.
\end{abstract}

\section{Introduction}

The Intensive Care Unit (ICU) is recognized as particularly suited to the implementation of AI-based monitoring tools [1]. The availability of a large quantity of data such as laboratory measures from blood samples, nurses annotations, routinely recorded parameters as drug infusion rates, up to continuously recorded vital signs such as the electrocardiogram (ECG), arterial blood pressure (ABP) and photoplethysmographic pulse (PPG) signal, provide an overwhelming amount of information that is quite difficult to be handled by clinicians. Lately, the use of AI tools, such as Machine Learning (ML), has proved the potential benefits of such techniques in the ICU environment [2,3]. Previous important studies have already developed successful models for the identification of false alarms in the ICU [4] and detection of atrial fibrillation [5] as well as the identification of hypotensive events [6].

To further improve ML models and standard statistical inferences, additional knowledge-based features could be inferred by appropriately devised mathematical models reflecting the subject's state from the available recordings. In this regard, Heart Rate Variability (HRV) measures, estimated from the ECG or photoplethismographic (PPG) signals by using knowledge-based cardiovascular models, provide the ability to track changes in the patients' autonomic control system [7] reflective of their cardiovascular state. Previous studies $[8,9]$ have analyzed the possibility of using Pulse Rate Variability (PRV) to replace specific HRV features under different conditions. However, to our knowledge, no other studies were conducted on the analysis of HRV parameters from surrogate signals, such as ABP or PPG, recorded from ICU patients. These patients are commonly identified as a critical population, often affected by different pathologies, chronic diseases and undergoing the administration of vasoactive agents, sedatives and mechanical ventilation procedure. Therefore, this study aims at investigating the validity of using different sources for estimating HRV measures, when dealing with ICU data.

\section{Methods}

\subsection{Data and Feature Extraction}

The study was performed using ECG, ABP and PPG waveforms extracted from the freely accessible Medical Information Mart for Intensive Care (MIMIC-III) Matched Waveform Database [10].

From the first 24 hours of recordings belonging to 79 distinct patients entered in the ICU, we extracted contem- 
poraneous, stationary and noise-free 10 minutes recordings of ECG, ABP and PPG waveforms (early). A second series of 10 minute recordings was extracted from those patients (50/79) that showed high quality recordings (late).

For each patient, after the identification of the R-peaks on the ECG using a Pan-Tompkins based annotator, we identified diastolic, pressure onset and systolic positions following the corresponding R-peak, from both ABP and PPG waveforms.

The pressure onset was defined as the point at maximum derivative between the systolic and the previous diastolic point. Together with the RR interval time series, we extracted pulse-to-pulse series from the successive time intervals of systolic, diastolic and onset fiducial points, from both ABP and PPG, thus obtaining six possible surrogates of the RR interval series, also called tachogram.

The selected HRV measures are: the average (AVNN) and the standard deviation (SDNN) of successive beat to beat intervals, the power in the low (LF: 0.04-0.15 Hz) and high frequency (HF: $0.15-0.45 \mathrm{~Hz}$ ) spectral bands, in both absolute and normalized units, $\mathrm{LFn}=\mathrm{LF} /($ tot power-VLF) and $\mathrm{HFn}=\mathrm{HF} /$ (tot power $-\mathrm{VLF})$, where VLF stands for power in very low frequency band (VLF: 0.004-0.04 Hz).

The power spectra of the tachogram and its surrogates are computed by parametric AR modeling of the RR time interval series of order chosen as the lowest providing residual white noise according to the Anderson's test, and complying with the Akaike's information criterion.

Additionally, the use of the matched subset allowed also the extraction of identifiers for mechanically ventilated and sedated patients, during extracted recording intervals, from the clinical part of the database.

\subsection{Statistical Analysis}

The proxy HRV measures extracted from the ABP/PPGbased surrogates were tested by comparing them with the corresponding ones extracted from the ECG-based tachogram (i.e. the gold standard), according to the following methods:

- Correlation Analysis: the correlation between parameters estimated from surrogates and the tachogram was computed and its p-value analyzed. Successively, after a linear fitting, the adjusted $r^{2}$ was evaluated.

- Statistical Distribution Analysis: between groups difference was tested through a Kruskal-Wallis test, after having assessed non-gaussianity through a Lilliefor's Test. Posthoc analysis with Bonferroni correction was conducted if a significant difference was found (Tab. 1). In order to investigate whether surrogates reproduce changes in the measures, a paired analysis through t-test or Wilcoxon signedrank test, according to the data distribution, was conducted between indices extracted from the second series of recordings (late) and the previous ones (early).

\begin{tabular}{|c|c|c|c|c|}
\hline & $E C G_{R}$ & $A B P_{O}$ & $A B P_{S}$ & $A B P_{D}$ \\
\hline $\mathrm{LF}\left[s^{2}\right]$ & 0.19 & 0.19 & 0.19 & 0.18 \\
\hline$\left(x 10^{-2}\right)$ & $(0.02-0.58)$ & $(0.03-0.58)$ & $(0.03-0.61)$ & $(0.03-0.62)$ \\
\hline $\mathrm{HF}\left[s^{2}\right]$ & 0.17 & 0.27 & 0.27 & 0.26 \\
\hline$\left(\mathrm{x} 10^{-2}\right)$ & $(0.03-0.80)$ & $(0.06-0.86)$ & $(0.05-0.94)$ & $(0.06-1.1)$ \\
\hline \multirow[t]{2}{*}{ LFn [nu] } & 0.27 & 0.24 & 0.23 & 0.24 \\
\hline & $(0.17-0.47)$ & $(0.14-0.39)$ & $(0.13-0.39)$ & $(0.12-0.40)$ \\
\hline \multirow[t]{2}{*}{ HFn [nu] } & 0.30 & 0.38 & 0.37 & 0.36 \\
\hline & $(0.2-0.48)$ & $(0.26-0.55)$ & $(0.25-0.54)$ & $(0.25-0.56)$ \\
\hline \multirow[t]{2}{*}{$\mathrm{LF} / \mathrm{HF}$} & 0.73 & 0.60 & 0.55 & 0.54 \\
\hline & $(0.40-1.80)$ & $(0.28-1.05)$ & $(0.25-1.03)$ & $(0.28-1.05)$ \\
\hline \multirow[t]{2}{*}{ AVNN [s] } & 0.74 & 0.74 & 0.74 & 0.74 \\
\hline & $(0.62-0.88)$ & $(0.62-0.88)$ & $(0.62-0.88)$ & $(0.62-0.88)$ \\
\hline \multirow[t]{3}{*}{ SDNN [s] } & 0.02 & 0.02 & 0.02 & 0.02 \\
\hline & $(0.01-0.03)$ & $(0.01-0.03)$ & $(0.01-0.03)$ & $(0.01-0.03)$ \\
\hline & $E C G_{R}$ & $P P G_{O}$ & $P P G_{S}$ & $P P G_{D}$ \\
\hline $\mathrm{LF}\left[s^{2}\right]$ & 0.19 & 0.24 & 0.24 & 0.21 \\
\hline$\left(\mathrm{x} 10^{-2}\right)$ & $(0.023-0.58)$ & $(0.055-0.66)$ & $(0.053-0.66)$ & $(0.057-0.63)$ \\
\hline $\mathrm{HF}\left[s^{2}\right]$ & 0.17 & $0.49 * * *$ & 0.37 & 0.35 \\
\hline$\left(\mathrm{x} 10^{-2}\right)$ & $(0.03-0.80)$ & $(0.21-1.43)$ & (0.14-1.19) & $(0.11-1.13)$ \\
\hline \multirow[t]{2}{*}{ LFn [nu] } & 0.27 & $0.16 * * *$ & 0.22 & 0.24 \\
\hline & $(0.17-0.47)$ & $(0.06-0.25)$ & $(0.10-0.30)$ & $(0.12-0.35)$ \\
\hline \multirow[t]{2}{*}{ HFn [nu] } & 0.30 & 0.36 & 0.38 & 0.41 \\
\hline & $(0.2-0.48)$ & $(0.23-0.48)$ & $(0.28-0.52)$ & $(0.28-0.55)$ \\
\hline \multirow[t]{2}{*}{$\mathrm{LF} / \mathrm{HF}$} & 0.73 & $0.38 * * *$ & $0.54 *$ & 0.57 \\
\hline & $(0.40-1.80)$ & $(0.24-0.60)$ & $(0.25-0.75)$ & $(0.29-0.88)$ \\
\hline \multirow[t]{2}{*}{ AVNN [s] } & 0.74 & 0.74 & 0.74 & 0.74 \\
\hline & $(0.62-0.88)$ & $(0.62-0.88)$ & $(0.62-0.88)$ & $(0.62-0.88)$ \\
\hline \multirow[t]{2}{*}{ SDNN [s] } & 0.02 & $0.02 *$ & 0.02 & 0.02 \\
\hline & $(0.01-0.03)$ & $(0.02-0.03)$ & $(0.01-0.03)$ & $(0.01-0.03)$ \\
\hline
\end{tabular}

Table 1. Median and interquartile range (in brackets) of each HRV measure coming from the tachogram and its surrogates (order of magnitude below the corresponding measure). Statistical difference from the tachogram is marked with $*(\mathrm{p}<0.05), * *(\mathrm{p}<0.01)$ and $* * *(\mathrm{p}<0.001)$.

- Bland-Altman Plots: eventually, in order to fully evaluate the agreement between methods in estimating the parameters of interest, a Bland-Altman analysis was carried out on measures extracted from each surrogate against the same measures extracted from the tachogram (Table 2).

\section{Results}

Correlation analysis shows that HRV measures computed from the ABP/PPG-based series are highly correlated with the respective measures computed from the tachogram. The lowest correlation (0.62) and adjusted $r^{2}$ (0.38) are found only for $\mathrm{LF} / \mathrm{HF}$ estimated from $P P G_{O}$, while a correlation greater than 0.7 and $r^{2}>0.52$ is found in all other cases. All HRV measures are significantly correlated with those extracted from the tachogram $(p<$ 0.001).

The median and the interquartile range computed for each measure are shown in Table 1. Of note, measures from $\mathrm{ABP}$ surrogates do not show any statistical difference with respect to tachogram. Conversely, LFn, SDNN and HF measures show statistical difference when estimated 

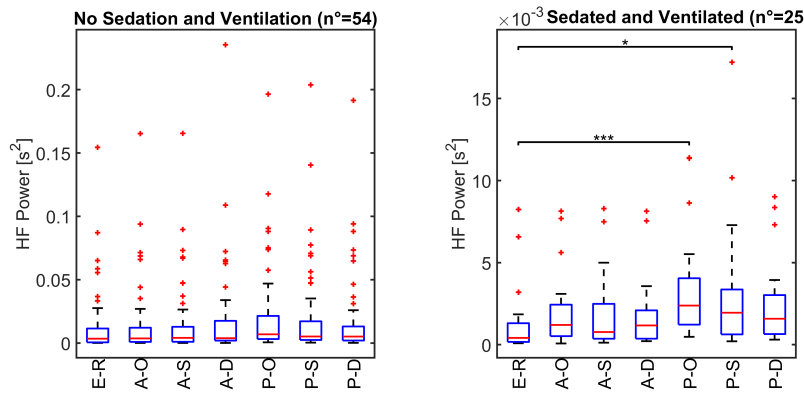

Figure 1. HF power estimated through RR intervals (E-R) and its surrogates, invasive blood pressure (A-) and photoplethysmogram (P-) onset $(\mathrm{O})$, systolic $(\mathrm{S})$ and diastolic (D) time interval series, for a sub-population of patients (25) under sedation and ventilation (right) and for those (54) who are neither sedated nor ventilated (left). Statistical significance between surrogates is indicated as follows: $* \mathrm{p}<0.05, * * \mathrm{p}<0.01, * * * \mathrm{p}<0.001$.

through $P P G_{O}$ while no significant differences are found for $P P G_{S}$ and $P P G_{D}$. In light of these differences, we investigated whether HF power shows similar behaviors when dividing the population into two groups according to major confounders for heart rate variability and particularly for HF measures, ventilation and sedation; the first group including subjects neither ventilated nor sedated and the other group with ventilated and sedated subjects. Results show significant differences only in the sedated and ventilated population and HF power in this last population results much lower than the other, as shown in Fig. 1.

Additionally, all indices extracted both from tachogram and surrogate series showed statistically significant difference ( $p<0.01$ for LF/HF and HFn estimated from PPGderived measures and $\mathrm{p}<0.001$ for ABP-derived series) when considering a subgroup of subjects that showed an increase from early to late conditions, and also significant differences $(\mathrm{p}<0.05$ for indices estimated from PPGderived measures and $\mathrm{p}<0.001$ for ABP-derived series) when a decrease is observed.

Table 2 shows the Bland-Altman 95\% limits of agreement between variability measures from surrogates and the respective ones from the tachogram.

\section{Discussions}

The presented study assesses whether PRV measures extracted from ABP and PPG beat to beat interval time series are good surrogates of HRV measures by looking at their agreement with those extracted from the tachogram.

Results in the correlation analysis show a very high correlation between HRV parameters from all surrogates and the ground truth. At the same time, results obtained looking at the distributions of the estimated measures with re-

\begin{tabular}{|c|c|c|c|}
\hline & $A B P_{O}$ & $A B P_{S}$ & $A B P_{D}$ \\
\hline $\begin{array}{l}\mathrm{LF}\left[s^{2}\right] \\
\left(\mathrm{x} 10^{-3}\right)\end{array}$ & $0.12 \pm 0.68$ & $0.23 \pm 1.0$ & $0.38 \pm 2.6$ \\
\hline $\begin{array}{l}\mathrm{HF}\left[s^{2}\right] \\
\left(\mathrm{x} 10^{-2}\right)\end{array}$ & $0.12 \pm 0.45$ & $0.18 \pm 0.82$ & $0.29 \pm 1.90$ \\
\hline LFn [nu] & $-0.05 \pm 0.15$ & $-0.05 \pm 0.18$ & $-0.05 \pm 0.16$ \\
\hline HFn [nu] & $0.05 \pm 0.14$ & $0.04 \pm 0.16$ & $0.04 \pm 0.12$ \\
\hline $\mathrm{LF} / \mathrm{HF}$ & $-0.33 \pm 0.99$ & $-0.35 \pm 1.09$ & $-0.30 \pm 0.91$ \\
\hline $\begin{array}{c}\text { AVNN [s] } \\
\left(\mathrm{x} 10^{-4}\right)\end{array}$ & $0.07 \pm 1.06$ & $0.15 \pm 1.03$ & $0.0076 \pm 1.51$ \\
\hline \multirow[t]{2}{*}{$\begin{array}{l}\text { SDNN [s] } \\
\left(\mathrm{x} 10^{-2}\right)\end{array}$} & $0.07 \pm 0.62$ & $0.10 \pm 0.73$ & $0.28 \pm 2.02$ \\
\hline & $P P G_{O}$ & $P P G_{S}$ & $P P G_{D}$ \\
\hline $\begin{array}{l}\mathrm{LF}\left[s^{2}\right] \\
\left(\mathrm{x} 10^{-3}\right)\end{array}$ & $0.70 \pm 2.20$ & $0.70 \pm 2.90$ & $0.40 \pm 1.60$ \\
\hline $\begin{array}{l}\mathrm{HF}\left[s^{2}\right] \\
\left(\mathrm{x} 10^{-2}\right)\end{array}$ & $0.61 \pm 2.06$ & $0.55 \pm 2.49$ & $0.31 \pm 1.46$ \\
\hline LFn [nu] & $-0.14 \pm 0.23$ & $-0.09 \pm 0.19$ & $-0.06 \pm 0.16$ \\
\hline HFn [nu] & $0.03 \pm 0.136$ & $0.04 \pm 0.15$ & $0.05 \pm 0.13$ \\
\hline $\mathrm{LF} / \mathrm{HF}$ & $-0.70 \pm 1.79$ & $-0.54 \pm 1.46$ & $-0.46 \pm 1.27$ \\
\hline $\begin{array}{c}\text { AVNN [s] } \\
\left(\mathrm{x} 10^{-4}\right)\end{array}$ & $0.48 \pm 3.0$ & $0.58 \pm 3.34$ & $0.33 \pm 2.10$ \\
\hline $\begin{array}{c}\text { SDNN [s] } \\
\left(\mathrm{x} 10^{-2}\right)\end{array}$ & $0.66 \pm 1.81$ & $0.48 \pm 1.99$ & $0.32 \pm 1.45$ \\
\hline
\end{tabular}

Table 2. Average difference and limits of agreement with 95\% confidence intervals of the Bland-Altman analysis. For each feature the order of magnitude is shown between brackets below the corresponding measure (when brackets are not shown, no rescaling is assumed).

spect to the reference ones show that, differences in the HF, $\mathrm{LFn}$ can be found for $P P G_{O}$ and differences in $\mathrm{LF} / \mathrm{HF}$ for both $P P G_{O}$ and $P P G_{S}$, indicating that caution has to be taken when using such surrogates. This results may be associated with marked respiratory and strongly influenced autonomic activities on PPG signal. This difference is not significant when diastolic successive points are used, possibly indicating these points as more stable (i.e. less affected by such effects) than previous ones. Respiratory incidence on HF power was investigated looking at only the sub-population of sedated and ventilated subjects, suggesting that HF power derived from $P P G_{O}$ may be influenced by such interventions (see Fig. 1). Indeed, it may be attributed mainly to the ventilation, i.e respiration, which strongly affects the PPG measurement, while the observed reduction in absolute HF power with respect to the not ventilated and not sedated population may be associated with the sedation procedure.

Results obtained in presence of changes in HRV indices suggest that the estimated measures are able to properly track significant changes as the HRV measures computed 


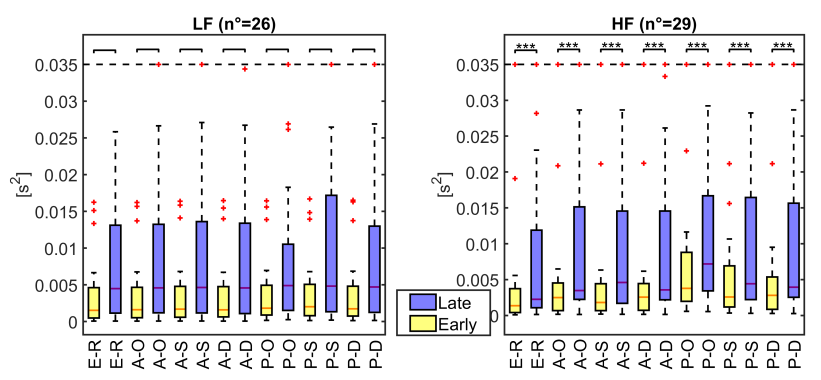

Figure 2. The figure shows increasing LF (left) and HF (right) powers, from early to late conditions, computed through RR interval (E-R) and its surrogates, invasive blood pressure (A-) and photoplethysmogram (P-) onset $(\mathrm{O})$, systolic (S) and diastolic (D) time interval series, for 26 and 29 patients, respectively. The trend remains significant $(p<0.001)$ for all surrogates in both panels.

from the tachogram do. An example can be seen in Fig. 2 , where subsets of subjects that showed an increase in LF and HF powers are shown.

Additionally, the Bland-Altman analysis shows a reasonably good overall agreement between surrogates and the reference measures with very small positive biases when estimating LF, HF, AVNN and SDNN indices. Conversely, a small negative (positive) bias for LFn (HFn) for surrogates from both ABP and PPG signals can be observed. This bias is always bigger for the latter than the former. Moreover, a strong negative bias can be observed in the estimate of LF/HF with $\mathrm{ABP}(\sim 0.3)$ and PPG surrogates $(\sim 0.5)$ with the previously observed trend. Confidence intervals show a small dispersion of all indices, with the only exception of LF/HF ratio which is strongly influenced by the use of surrogates. These results suggest that the use of these surrogates may underestimate (overestimate) the sympathetic (vagal) activity on the autonomic control in modulating the heart activity. Finally, diastolic surrogates from PPG recording showed smaller biases and confidence intervals than other PPG-derived indices.

Our findings agree with those obtained by previous studies with subjects under different conditions as rest, tilt and mental stress $[8,9]$ and expand them toward a population with a high variability and in very unstable and critical conditions as ICU patients are.

In conclusion, despite some accountable differences between surrogates and original HRV measures, we can consider ABP-derived HRV measures as good surrogates of autonomic activity estimated by ECG in ICU patients while caution has to be taken for PPG-derived ones, among which those extracted from the diastolic time intervals $\left(P P G_{D}\right)$ appear to be less affected by ventilation and sedation procedures.

Future studies will deeply investigate the role of differ- ent treatments and procedures on HRV surrogates.

\section{References}

[1] Hanson CW, Marshall BE. Artificial intelligence applications in the intensive care unit. Critical Care Medicine 2001;29(2):427-435. ISSN 00903493.

[2] Johnson AEW, Ghassemi MM, Nemati S, Niehaus KE, Clifton DA, Clifford GD. Machine learning and decision support in critical care. Proceedings of the IEEE 2016; 104(2):444-466.

[3] Vellido A, Ribas V, Morales C, Ruiz Sanmartín A, Ruiz Rodríguez JC. Machine learning in critical care: stateof-the-art and a sepsis case study. Biomedical engineering online Nov 2018;

[4] Plesinger F, Klimes P, Halamek J, Jurak P. False alarms in intensive care unit monitors: Detection of life-threatening arrhythmias using elementary algebra, descriptive statistics and fuzzy logic. In 2015 Computing in Cardiology Conference (CinC). 2015; 281-284.

[5] Datta S, Puri C, Mukherjee A, Banerjee R, Choudhury AD, Singh R, Ukil A, Bandyopadhyay S, Pal A, Khandelwal S. Identifying normal, af and other abnormal ecg rhythms using a cascaded binary classifier. In 2017 Computing in Cardiology (CinC). 2017; 1-4.

[6] Hatib F, Jian Z, Buddi S, Lee C, Settels J, Sibert K, Rinehart J, Cannesson M. Machine-learning Algorithm to Predict Hypotension Based on High-fidelity Arterial Pressure Waveform Analysis. Anesthesiology The Journal of the American Society of Anesthesiologists 10 2018; 129(4):663-674. ISSN 0003-3022.

[7] Task Force of the European Society of Cardiology the North American Society of Pacing Electrophysiology. Heart rate variability. Circulation 1996;93(5):1043-1065.

[8] Pernice R, Javorka M, Krohova J, Czippelova B, Turianikova Z, Busacca A, Faes L, Member IEEE. Comparison of short-term heart rate variability indexes evaluated through electrocardiographic and continuous blood pressure monitoring. Medical Biological Engineering Computing 2019;57(6):1247-1263. ISSN 1741-0444.

[9] Schäfer A, Vagedes J. How accurate is pulse rate variability as an estimate of heart rate variability?: A review on studies comparing photoplethysmographic technology with an electrocardiogram. International Journal of Cardiology 2013;166(1):15 - 29. ISSN 0167-5273.

[10] Johnson AEW, Pollard TJ, Shen L, Lehman LwH, Feng M, Ghassemi M, Moody B, Szolovits P, Anthony Celi L, Mark RG. Mimic-iii, a freely accessible critical care database. Scientific Data 2016;3(1):160035. ISSN 2052-4463.

Address for correspondence:

Maximiliano Mollura

Via Ponzio 34/5, 20133 Milano, Italia

maximiliano.mollura@polimi.it 